\title{
The «Hominescent» Body in Michel Serres Thinking
}

\section{Rignani $0^{*}$}

University of Florence, Italy

*Corresponding author: Dr. Orsola Rignani, Work: Via Laura, 48, 50121 Florence, Italy Home: Via Curiel, 4, 43123 Parma, Italy, Tel: 393383350263; Email: orsola.rignani@unifi.it

\section{Research Article}

Volume 1 Issue 1

Received Date: July 20, 2018

Published Date: August 10, 2018

DOI: $10.23880 /$ phij-16000101

\section{Abstract}

My contribution examines and discusses Michel Serres idea, expressed in the eighties, of the «reappropriation» of the senses (and body) in the way of aesthetics as a «reunified» discourse on the data of sensations and fine arts, and his idea expressed in the nineties of the metamorphism and mimicry of the body, reading them as emergencies of his recent idea of the new «hominescent» body; in order to focus on the latter meanings and implications. First I will examine, precisely in the horizon of Serres philosophy of hominescence, Serresian proposal «to return» to the senses and body in the perspective of aesthetics understood as said. I will then consider Serres' theming of body metamorphism and mimicry, within which the senses are considered to act as body's mimicry control. These steps will lead me, hopefully, to shed light on the implications of Serres' recent idea of the «hominescent» body.

Keywords: Michel Serres idea; Hominescent Body; Senses; Theming of body; Metamorphism; Mimicry

\section{Introduction}

My essay examines and discusses Michel Serres' idea, expressed in the eighties, of the «reappropriation» of the senses (and body) in the way of aesthetics as a «reunified» discourse on the data of sensations and fine arts, and his idea, expressed in the nineties, of the metamorphism and mimicry of the body, reading them as emergences of his recent idea of the new «hominescent» body [1-4]; with the aim of focusing on the meanings and implications of the latter.

\section{The «reappropriation» of the Senses (and body)}

Firstly, I consider Serres's proposal of the «return» to the senses and body in the way of aesthetics interpreted as said, in the perspective of the Serresian philosophy of hominescence.

In the beginning of the twenty-first century, Serres formalised and explained the idea that humanity has been searching for and (why not?) finding its own way to develop «new» forms of understanding itself, its environment and changes (socio-economic, political, cultural, techno-scientific etc.) which have occurred in recent decades. To expound upon his theory, he coined the inchoate neologism hominescence (new «beginning», «differential» of «(self) hominisation», set of bifurcations, which are seen in the process of redefinition/recharacterisation of the human condition), thematising the «emergence» of unprecedented relations with the body, the world and at a collective level (Serres [2001]: 21) [1]. 


\section{Philosophy International Journal}

That is, on the «subjective» level there emerges a «liberation» of/from the body (body free from pain, from sickness, from the ancestral "subjection» to the soul, mind, etc., which «gets rid», «objectifying» them, of its organs and functions, healthy and long-lived; new links with the body and new roles of corporeality; the body is no longer a «constraint»); then on the «objective» level emerges an emancipation from dependence on «things» (man becomes naturans of a new nature produced by him and which produces effects on him); and on the «collective» level emerges a decoupling of social relations and communications from the spatial conditions (it inhabits a space «without distance», qualitative, in which "concentration» is giving way to "distribution» and in which, with information and communication technologies, the «connective» is replacing the «collective») [4].

Thus it is so that in this expression/declination of the dimensions of hominescence Serres ranks first the «emergence» of links without known equivalents to body (ibid.), identifying and highlighting its crucial role in the «hominescent» breakthrough: the advent of a body free from the contraintes (constraints) that inhibited it marks a new human condition; the objective bodily changes elicit in man a perception/awareness of them, then a rethinking of the body itself and its role and therefore a theming/management of the novelties: a «revolution» from which, as such, is to be invested the être-aumonde (being-in-the-world) as a whole.

Of this idea of the "hominescent» body I seem, after also collecting internal references in Serres' texts (Serres [2001]: 58-59) [1], to be able to read as the emergences the Serresian proposal of the eighties of the "return» to the senses (and thus to the body and the «things») and the idea, proposed at the end of nineties, of metamorphism and bodily mimicry (I feel I should also trace, lato sensu, the theme of hominescence even further back, to the sixties, with the theming of historic transition from the kingdom of Prometheus to that of Hermes, i.e. from energy to information) [5-9]. Through such a reading, however far it is from linearism not at all adequate to a reflection and a work such as those of Serres from the hypertextual, circumstantial, and pluri-dimensional physionomny, I hope therefore to be able to derive (further) views on the traits of the «hominescent» body.

Thus I now turn to consider Serres' proposal/idea, formulated in The Five Senses, of the "rediscovery» of the senses (referred to as «the skin, hearing's pinna, the two non-verbose tongues of flavors and kissing, the visit on the move of world's lanscapes» (Serres [1999]: 8)) [10] in the face of what he believes as a succession, from ancient to contemporary times, of the «dominance» of language, science and codes (in a critical comparison specifically with cognitivist and analytical perspectives; with what he considers the tendency of «reduction» of the sensible to word etc.); in the whole context of what he gathers and thematises as the passage from Prometheus to Hermes, i.e., as mentioned, from a culture and a society dominated by production to one increasingly dominated by the transmission and distribution of messages (Serres [2015]: 11-13, 47-49, 201-202, 213-215) [11].

The convinction of the «loss of the world», of the acosmism of science and philosophy thus causes Serres to go on an operation of the "rescue» of the senses and the world, that is a revisit/reevaluation of the sensory universe (on the way to a rebalancing of the sensible and intellectual), as well as to «look at» man from the point of view of the world, and then to the "reinsertion» of the world in the same philosophical discourse. In the name of aesthetics that «has only one sense» (Serres [1985]: 130) [12], that is, a discourse on the data of sensations and fine arts («re-united» with respect to the separation often drawn between these two meanings): «the world displays itself as beautiful» (ibid.); the data, that is the "gifts» of the world, «offer» themselves in their sensory gratuity, in a relationship which combines beauty and life(«we need beauty in order to live» (ibid.)).

In the perspective of such «sensible aesthetics, the unified field of beauty» (ivi, 215) indeed, Serres then launches his proposal of the «return» to the senses, which is informed after all by the intention of a distancing from what he regards as the privilege traditionally granted by philosophy to sight to the detriment of hearing, and even more of touch and smell; from abstraction as «cutting» of the sentient body («analysis»), suppression of taste, smell and touch (ivi, 26). Privilege and abstraction which he believes can be counterbalanced by the idea of a «slip» from sight to touch and towards the skin (which in itself implies touch). Skin, within which he first identifies the inextricable mingling of soul and body, rejecting the idea of a fixed «position» of the soul itself in the body, to which he opposes the belief that the soul protrudes in «contingencies» (in the sense of «common tangency» (ivi, 80 ), i.e. contact) of the body with itself and with the world. The belief that the soul is «inscribed» in going and coming of subjectivity on the surface of the skin as the remainder of its "contingencies», the play of light and shade of the same subjectivity and objectivity (ivi, 21-26).

Skin, topological variety (ivi, 61) (we must keep in mind that the topology, the science of the neighbourhood, of the intervals, of the borders, indeed of variety, of the 


\section{Philosophy International Journal}

open and closed etc., is an organon of the Serresian approach to sensing), each "place» of which Serres therefore deems to constitute a different mix: the simple elements that we are accustomed to see in nature are originated from mixtures of topological variety modelled as the skin itself (ivi, 28).

In it Serres moreover locates the mixing of the flows coming from the sensory organs, in the manner of a sensorium commune; he considers it to form a variety of mingling senses, to exert sensibility (ivi, 52) in its fullness, to receive the specificity of sensory organs: a sensorium commune, a «synthesis» of the five senses (ivi, 54), a common sense of all senses which acts as a bond, a bridge, a passage between them (ivi, 70); the first approximation to mélange (mixture) (fluid, floating, varied and contingent, favouring the merger (ivi,80, 81)), poorly tolerated, on the other hand, by sight, more precisely inclined in analysis and differentiation.

Serres therefore believes that the skin enters into the things of the world and encourages their blending, implicates all the sensory organs, forming the «model» on the basis of which every sense, bouquet of the other senses and mixture of the blending modes, can implement this synesthesia. In short, he considers it the thing in the world that best takes part in the body (ivi, 70).

Thus it is that, for the mélange (mixture) and the "principle» of "contingency», the skin (as well as touch) can be but a keystone of Serresian philosophy of «mingled bodies» (of which The Five Senses, subtitled A Philosophy of Mingled Bodies (I) and not by chance opened with a discussion on skin, precisely presents itself as a crucial text), which is a reflection that, in general terms, distancing itself from intuition, from the solid rigor of sight, from analysis, from dualisms, advances toward the fluid, the aerial, meeting turbulence, toward a thought on fusion, on confluence, on the mélange (mixture) and thus on «mingled bodies».

Getting closer to this mélange (mixture);grasping it, the state of things in its mingling, topological multiplicity of veils, weaving of threads (extra the «simplifying» idea of truth as discovery, and instead «unveiling» in the sense of patiently following the arrangement of the veils, the close spaces, the depth of their interweaving)(ivi, 82):according to Serres, this can happen especially in the area of topological tact, in the skin, or in any case in the rich, vivid, complex experience of the senses, which are after all the blending of the body, the ways through which the body mixes itself with the world and itself, exceeds its own borders. They are, in other words, a way of being in the world (and not in front of it to see it) essential for knowledge; understood, the latter, in the perspective of the recognition that «I mix with the world which mixes with me» (ivi, 80), that is, in other words, as to place oneself within the mix of things (ibid.).

Thus, this Serresian excursus through the qualitative spaces of sensory topology proceeds through skin-touch, hearing, smell-taste, sight (not so much according to a hierarchical order of the senses themselves, even though redesigned/renewed, but rather according to a recombination of them to discover their operation, to revise the traditional attributions of superiority or inferiority as well as their separation).

This excursus captures hearing as an ability/function of transformation from «hard» (energy etc.) into «soft» (information etc.); endowed with a mingled nature, involving the whole body (the skin and various organs); as exposure to things and integration of them together (ivi, 85-151).

It captures taste and smell (senses often overlooked or poorly treated) to be irreducibly combined, in their «open up» the "second mouth», that of taste that opens up to grace and to the «gifts/data» of the world (outclassed and anesthetised by the "first mouth», that of language); capturing the wisdom as taste finally called appropriately and sagacity as smell returned to its cognitive dignity (ivi, 152-235).

It captures sight in its negative meaning as divisional attitude against the mélange(mixture)that is returned by the other senses; however identifying a rehabilitation of it in terms of «visit», i.e. moving vision or going to see the landscapes of the world. If, in fact, sensible can be understood in a similar meaning to that of adjectives with the same "ending» and in this sense expresses an ever possible change of direction (open like a star or almost closed like a knot, it has all the senses, it is mobile, and it is an exchanger), it allows you to speak precisely of vision as «visit» and «to visit», that mean in the first place to look and to see, but with the addition of the idea of movement: moving to see; sight as «visit», «sense» which implicates direction changes, deviations, turning and intersections. Thus, it ends up reuniting with itself the deviation, the «deflective» character of the whole sensory experience: the sensible «holds together», as a heat exchanger node, all the senses, all sizes and contents, i.e. precisely the different «lands» through which the «visit» passes; «visit» which then, for its part, explores all the senses of the sensible implicated in the latter's node (ivi, 


\section{Philosophy International Journal}

236-310). And then only the «visit» (without exactly «dividing»/«dissecting» the sense of the word «visit») allows you to see the compact capacity of the senses (over undue separations between them).

Senses, therefore, such as «to visit» the world: the body, in the process of knowing, through the senses, «visits»; it «exceeds» itself; it «builds» itself through them, as they are conditions of its possibility of being «beyond» itself; it comes out by itself «in all senses» living with the sensible in a perpetual exchanger («circumstance», i.e. all around the substance, equilibrium plus change), passing in a multiplicity and thus becoming a mongrel, a hybrid(«I feel therefore I pass, a chameleon in a gaudy multiplicity, I become a half-caste, quadroon, mulatto, octoroon, hybrid» (ivi, 307)); ground for hybridisation and, as such, neuralgic place of knowing.

The Serresian «rediscovery» of the senses («under» the «anaesthesia» of language, the «hegemony» of sight etc.) therefore goes to point out on the whole the idea of a body to which, in its entirety, the knowing «subject» (no longer concentrated in a «place») extends; then the idea of a knowing body, facing a new horizon: one knows or understands through the skin, subtle as the iris or the pupil, which are in turn subtle like intuition; one understands or knows through wisdom, taste finally named correctly, and through sagacity, smell finally returned to its cognitive dignity; as well as, on the other hand, one knows or understands through muscles, breathing, running, jumping, dancing etc. The knowing «subject» then goes back to his «real» residence that is precisely the body, occupying it all, home to a comprehensive knowledge based on the expertise of the senses and given to the limbs. A body «subject», flexible, bright, transparent: «thinking».

I thus believe that, in Serres, the "return» to the world through the senses, that is the «rediscovery» of the senses and the world in the way of aesthetics as a discourse together on sensation and beauty, points out the «hominescent» change, the reconnaissance of whose corporeal dimension has, as I believe, the trigger as well as a neuralgic element in the same «rediscovery» of the senses.

It thus starts to exit the objective change in the body as a whole (it's becoming healthy, long-lived etc.); the human perception/awareness of this change; the change in the perception of the body and the advent of new relations with it. Namely the «rediscovery» of the body was thinking, knowing etc., the start of a dimensional relationship with it (as companion, double of the man), and therefore the progressive formal notice of the idea of the body reified, object, instrument. Just as the theming of the change itself, namely the idea that these changes in and related to the body are/may is of revival to a new «subject», to which new data is given, on the way to a full-bodied man's relationship with the world.

\section{The Metamorphic and Mimetic Body}

If in the Serresian proposal, of the eighties, of the «recovery» of the senses and the body I read emergences of the idea of the new «hominescent» body, I also find them in the proposal, of the nineties, of metamorphism and bodily mimicry. Idea, however, that seems to revive and, so to speak, articulate the perspective shown in The Five Senses. If, in fact, in this work the body is considered to be in constant exchange with the world, marked by «circumstances» and continually returned to them and to the exercise of the senses that work it like a landscape, land of knowledge, of a knowledge based precisely on the competence of the senses themselves and granted to the limbs, in Variations on the Body the knowledge itself, of which the senses are not considered the only source, is considered to emerge largely by imitation that the extraordinary plasticity of the entire body makes possible. (The title Variations on the Body, I purposely note, is not random and is not taken only by the musical context: Serres thinks of the body with its metamorphoses as an invariant through the variations of flexibility, imitation etc., drawing on a black box that consists precisely of invariants through variations, of the topology, as well as the mechanics of fluids, information theory etc.).

In Variations therefore Serres further articulates/declines compared to The Five Senses the idea of the flexibility of the body, thematising metamorphism of the latter, i.e. detecting, in the relationship between the body «unifying» the senses and the world, the plasticity of the body itself. And therefore, its potential existence, namely the potency as its «essence», for which it «bends», «curves», «adapts». It plays outside the equilibrium, and in this sense bifurcates, it invents in a change of direction, it breaks, in the obliquity, the symmetry -I note here in passing the assumption by Serres of Lucretius' theory of clinamen as a reference for his own interest for the bifurcation, point without dimension from which everything changes direction, break of symmetry, invention, beginning («hominescent», «incandescent» etc.) (Serres [1977], [2014]: 79-80, [2015]: 76-89) $[13,14,11]$, it circumvents the laws of statics, establishing its own structure within the instability, the potential: it exists in potency in all senses (Serres [1999]: 46) [10]. In 


\section{Philosophy International Journal}

fact, in Variations the constellation of potency protrudes, with a slippage of the thinkability of man from the definition in the skew, in the asymmetry, i.e. in excess and indefiniteness dimension (Serres [2003]: 366) [2]. Which emerges of man himself with/in the body, emerging in its so-called maximisation of the possibility... metamorphism.

If movement, change, or exchange are necessary for the definition of life and therefore apply also to the body, Serres, however, does not consider them sufficient to account for the sudden wastes, unpredictable from the genetic point of view, made by movement, by change, by body exchange: abandoning a constellation of forms, the body assumes another, distinguishing itself from nonhuman living beings for that process, hardly predictable by the sciences of life, which is metamorphism. All living beings live of metabolism; man lives (also) of metamorphism, endlessly multiplying gestures and expressions, so much that his body, open to an immense range of postures, signs etc., is precisely indefinable in substantial terms and thinkable rather in the potential, in the virtual (Serres [1999]:51-52) [10].

In divergence with respect to the philosophical tradition that «took the floor» and has cut dancers, mimes, athletes, children etc. off from speaking (that's those from the body equipped with the maximum degree of freedom, ductile to each type of transformation) thus throwing the same body and the extraordinary proliferation of its forms in the shadows, Serres therefore «reconvenes» the rank of the dancers, mimes, athletes, children and collects/thematises the infinite metamorphic and mimetic capacity of the body itself which «can do many things that astonish the mind» (ivi, 54).

Mimetic process of which, furthermore, Serres detects a «first» metamorphosis (the neotenic, «incandescent» as a condition of openness to otherness; «incandescence» like incohatio, beginning and also non-ownership, which «marks» the availability to the transformation) [2] in which the body crosses all the species, since man, by imitating them, synthesises them exactly. And a «second» metamorphosis in which the arrest of the vital flow stiffens the man himself, aged, in a «particular animal», a «specialised» one. The resistance, the opposition to the «second» metamorphosis, however, requires that man remains «deskilled», available at any kind of simulation as long as it is reversible; in virtuality, flexibility, and «incandescence».

In bodily metamorphosis Serres captures then an understanding accessing the basic natures (minerals, plants, animals), entering in the dynamics of mélanges(mixture), for which the "confluence» is to be the path of knowledge, which, without this mélange(mixture), without the simulation and metamorphosis that it produces, could hardly be carried out precisely (Serres [1999]: 55-60) [10].

Possibility of the body then «to produce a thousand possible metamorphoses» (ivi, 61) («omnivalent substratum for all these transformations»(ibid.)), plasticity which makes possible imitations from which much of the knowledge comes; (knowledge) of which the senses are not considered the only source.

One of Serres' concerns in this regard, in Variations, is, in fact, to decline the idea of the knowledge of the body (such as subjective genitive), that is, the idea of a body by its presence and cognitive function, alternatively to those which he considers proposals of a genesis of «bodiless knowledge» (sensism, logical empiricism etc.), whose «from the senses toward understanding» procedure ends up, in his view, obscuring the rest of the body itself, or rather reducing it to the function of merely carrying the five peripheral terminals (ivi, 69).

Thus arriving to affirm that there is nothing in knowledge that has not been first in the entire body (ivi, 70), whose gestural metamorphoses, mobile postures and very evolution itself mimic everything that surrounds it, Serres points out, therefore, that the body is both gestural and receptive, and therefore more active than passive, skeletal, muscular... bearer of the five senses, but with different functions from that of conveying external information towards a «processing centre». A body, therefore, that is not merely a vehicle of the senses, but rather provided with cognitive functions neutralized by the couple sense-understanding, «whereas imitation, on the contrary, implies sensory activity» (ibid.).

Sensory activity, senses which Serres in this perspective is then to consider as, normally, the control of the mimicry of the body, and as, less frequently, routes towards cultural refinement (development of a refined taste, a smooth smell, a velvety touch, a vision sensitive to shades, a musical ear etc.) or to the mastering of an art (ivi, 71-72).

Thus, in Variations, Serres, as I have mentioned, further expounds upon the idea expressed in The Five Senses of the whole body in its plasticity, as the ground of knowing, in which the senses, not the only source of all knowledge, "converge», normally, in the dynamic of metamorphism of the body; i.e. the idea of a body in 
motion, which expresses itself less in the sensibility than in gymnastic exercises, in dance, in gestures etc.

Therefore, body plasticity, as a whole; which, I repeat, makes possible imitations from which the majority of knowledge emerges. Mimicry, then, as a source of knowledge: «we don't know anyone or anything until the body takes on its form, its appearance, its movement, its habitus, until the body joins in a dance with its demeanor» (ivi, 70-71); thus, body schema is acquired, it is deposited in the memory, it is refined. "To receive», «to issue», «to store» and «to transmit» are the deeds of the "expert» body. The act of miming, then, gives rise to the reproduction, the representation and the virtual experience, functions in which Serres detects the priority of the same body, thrown into oblivion over the centuries by the different methods of memorisation and writing (tablets wax, parchment, printing etc.)(ivi, 71).

Body which Serres identifies as the «subject» of knowledge (understood, the latter, in opposition to rationalist ideal of clarity as a condicio sine qua non of learning -one cannot learn what one does not understands-, as knowledge which occurs in the «opaque» -one learns many more things than those that one comprehends-): «the body remembers and forgets, it is capable of more or less what it believes itself capable, does better or worse than it knows it can do, does not know and knows at the same time» (ivi, 77). If, therefore, Serres identifies in «taking» (essentially as to «con-tact»), in «learning» (as to mix with things) and in «understanding» the learning stages in succession, then he perceives the body such that «knowledge sinks into it and from it re-emerges. Hidden in the shadow, the body slowly assimilates the simulated» (ivi, 78).

Gestures don't need to be repeated many times for the body to make them its own; in bones and muscles it incorporates complex chains of postures so easily that it loses the very memory of this complexity; then, suddenly, almost unconsciously, it reproduces these sequences of positions more rapidly than it does not assimilate them: it «mimics», «deposits», «recalls». It's the first base of human cognition, then, in the form of «incarnate» memories; the primary support of memory. The mimicry thus emerges as a start-up of knowledge and the body as a set of memories imprinted and integrated by a long and patient mimetic adaptation process; of which, in fact, we tend to lose the memory, forgetting the metamorphic work of memorization (ivi, 106-108).

The metamorphoses of the body and the mimicry of learning are made possible, as I have said, by the flexibility, in accordance with the idea of a «préposé» or «re-posé» body, prepositional-one must not forget the importance of prepositions in the thought of Serres and his «philosophy of prepositions», i.e. precisely invariant through the variations of plasticity, of imitation, of life itself etc. In order to preserve this invariance, the same body exchanges energy and information with its environment, that is «subjectifies» and «objectifies». «Objectifies» in the sense that, through a process of «casting off», it «loses»; the organs are «deskilled», i.e. they are emptied of their forms and functions to pour them outside; limbs, their gestures, and their movements «leave» the body to form «tools similar to them, but cast off from them» (ivi, 118); memory pours its contents onto pages, books, libraries; imagination leaves its icons on paper, canvas, screens etc.; intelligence puts its operations into the calculator etc. With this «lightening» of the body, found in «casting off» moments, Serres sees, on the other hand, often coincide the invention, the new: when, for example, the press has relieved man from the task of remembering, the experimental science saw the light etc. In such «casting off» corresponds, for Serres, vice versa the «learning», «subjectification» process that slowly traces back the path of inventions: come out of the body through strokes of genius, the same inventions return there in familiar, formative context etc. (ivi, 118-121). Thus it is that imitation, training, formation, as mentioned, adopt new gestural sequences in the body, and the latter adopts them sometimes to the point of forgetting them to better reproduce the patterns or to inventively vary on them. So through «assimilation» the body «subjectifies» things and movements; objects, for their part, are «subjectified» through nourishment, inspiration, imitation and learning, in a continuous exchange, as I said, between the environment and the body itself, in which the latter precisely preserves invariance: in the dynamics of «subjectification» and "objectification» life is maintained and developed, and, especially, culture is originated.

Dynamics in which, after all, protrudes body's «incandescence» [2] mentioned above: having externalised organs and/or functions, the body despecializes, that's becomes «incandescent», i.e. undifferentiated, white; and, in/for this, condition of the technical, cultural, linguistic differences, i.e. «transcendental» (condition of possibility), culturally totipotent (all-powerful, potentially contains all the cultural varieties). Totipotence then of the body and so of the man, this «new subject», the "Incandescent», in which the body itself, in its «incandescence», emerges in its cruciality. That's in its dimensional value (to be a body): 


\section{Philosophy International Journal}

«transcendentality», virtuality, «prepositionality» (in it, with it and through it).

\section{The «hominescent» Body}

With his project of a «new» culture, lato sensu, for a «new» humanity, Michel Serres gives us a new anthropology in admission of the possibility of even building a discourse, at the time however in a nascent state, on the human being; a new discourse on a «new» human being, in which the body takes a crucial role. Which, as I suggested at the beginning and which $\mathrm{i}$ now feel can be confirmed, emerges most clearly in the light of the same ideas/reflections on the body that he proposed in the eighties and nineties. The body, which came out from The Five Senses as constructed and reconstructed through the senses, thinking, neuralgic terrain of a knowledge founded on the competence of the same senses and given to the limbs, «subject», flexible etc., and jutting from Variations as metamorphic, mimetic, knowing, omnivalent, totipotent etc., therefore I think indicates the new «hominescent» body in its emergence, first of all, in neutralization on the plain of being and in reactivation in terms of the modes (extra fixity, factuality etc.), that's as «capable», «incandescent». Thus in the orbit of virtuality, of possibility, of «prepositionality». A «hominescent»body, «transcendental»: a «cultural prow», which is able to open new horizons of humanity; and therefore, a double, a companion of man, bearer of the senses and intelligence, in constant exchange with the world, a land of transformation, of transmutation («external»/«internal», energy/information etc.), itself in transformation, in transmutation. A dialogic space of construction of the human in the exposure and opening [15-18].

In conclusion, the idea of the «reappropriation» of the senses and the body, in the way of aesthetics as a discourse on the data of sensations and the arts, and the idea of metamorphism and mimicry of the body seem to me to be emergences of the new «hominescent» body. Which emerges on the whole as transitional, under construction, in possibility; ground/fly wheel of knowledge and invention that dimensions the human, in the exchange, in the mélange (mixture); dimensional/dialogical space of anthropopoiesis, which always opens new horizons of humanity itself, in a perspective of continuity with the other-outside-the-man [19-21].

So: new body, perception of this novelty, new perception of the body, theming of these novelties, in the perspective of the «hominescent» idea. That's to say the idea of a human condition «subjective», «objective» and «collective» transformation, which, as mentioned, implies that man produces himself what was previously given: the body in fact, life, death, nature.

According to «hominescent» idea, therefore, man, for the first time in his history, no longer inherits his condition, but starts to produce it himself, entertaining precisely new relationships with his body, with the world and with his similar.

\section{References}

1. Serres M (2001) Hominescence. Le Pommier, Paris.

2. Serres M (2003) L'Incandescent. Le Pommier, Paris.

3. Serres M (2009) Temps des crises. Trans. by Feenberg-Dibon AM, Times of Crisis. What the Financial Crisis Revealed and How to Reinvent our Lives and Future. Bloomsbury Academic, New YorkLondon.

4. Serres M (2012) Petite Poucette. Trans. by Smith DW, Thumbelina: The Culture and Technology of Millenials. Rowman \& Littlefield International, London.

5. Serres M (1969) Hermès I, Communication. de Minuit (Edn.), Paris.

6. Serres M (1972) Hermès II, L'Interférence. de Minuit (Edn.), Paris.

7. Serres, M (1974) Hermès III, La Traduction. de Minuit (Edn.), Paris.

8. Serres M (1977a) Hermès IV, La Distribution. de Minuit (Edn.), Paris.

9. Serres M (1980) Hermès V, The Northwest Passage. de Minuit (Edn.), Paris.

10. Serres M (1999) Variations sur le corps. Trans. by Burks R, Variations on the Body. Univocal, Minneapolis, 2011.

11. Serres M (2015) The lame southpaw: the power of thought, Le Pommier, Paris.

12. Serres M (1985) Les Cinq Sens. Trans. by Sankey M, Cowley P, The Five Senses. A Philosophy of Mingled Bodies (I). Continuum, London-New York, 2008. 
13. Serres M (1977b) La naissance de la physique dans le texte de Lucrèce. Trans. by Hawks J. The Birth of Physics. Clinamen Press, Manchester, 2000.

14. Serres M (2014) Pantopie: from Hermes to Petite Poucette. Le Pommier, Paris.

15. Assad ML (1999) Reading with Michel Serres. An Encounter with Time, State University of New York Press, Albany.

16. Connor S (2004) Topologies: Michel Serres and the Shapes of Thought. Anglistik 15: 105-117.

17. Connor S (2005) Michel Serres's Les Cinq Sens. In: Abbas N (Ed.), Mapping Michel Serres. University of Michigan Press, Ann Arbor, pp: 153-169.
18. Crahay A (1988) The Mutation of the Cogito: Genesis of the Transcendental Objective, De Boeck, Bruxelles.

19. Polizzi G (1990) Michel Serres, For a philosophy of mixed bodies. Liguori, Napoli.

20. Rödel P (2016) Michel Serres, la sage-femme du monde. Le Pommier, Paris.

21. Serres M (2016) Darwin, Bonaparte and the Samaritan - A philosophy of history. Le Pommier, Paris. 